\title{
Diabetes risk and amino acid profiles: cross-sectional and prospective analyses of ethnicity, amino acids and diabetes in a South Asian and European cohort from the SABRE (Southall And Brent REvisited) Study
}

\author{
Therese Tillin • Alun D. Hughes • Qin Wang • Peter Würtz • \\ Mika Ala-Korpela • Naveed Sattar • Nita G. Forouhi • Ian F. Godsland • \\ Sophie V. Eastwood $\cdot$ Paul M. McKeigue $\cdot$ Nish Chaturvedi
}

Received: 7 November 2014 / Accepted: 15 January 2015 / Published online: 19 February 2015

(C) The Author(s) 2015. This article is published with open access at Springerlink.com

\begin{abstract}
Aims/hypothesis South Asian individuals have an increased risk of diabetes compared with Europeans that is unexplained by obesity and traditional or established metabolic measures. Circulating amino acids (AAs) may provide additional explanatory insights. In a unique cohort of European and South Asian men, we compared cross-sectional associations between AAs, metabolic and obesity traits, and longitudinal associations with incident diabetes.

Methods Nuclear magnetic spectroscopy was used to measure the baseline (1988-1991) levels of nine AAs in serum samples from a British population-based cohort of 1,279 European and
\end{abstract}

Electronic supplementary material The online version of this article (doi:10.1007/s00125-015-3517-8) contains peer-reviewed but unedited supplementary material, which is available to authorised users.

T. Tillin $(\bowtie) \cdot$ A. D. Hughes $\cdot$ S. V. Eastwood $\cdot$ N. Chaturvedi

UCL Institute of Cardiovascular Science, 170 Tottenham Court

Road, London W1T 7HA, UK

e-mail: t.tillin@ucl.ac.uk

Q. Wang $\cdot$ P. Würtz $\cdot$ M. Ala-Korpela

Computational Medicine, Institute of Health Sciences, University of

Oulu, Oulu, Finland

Q. Wang $\cdot$ P. Würtz $\cdot$ M. Ala-Korpela

NMR Metabolomics Laboratory, School of Pharmacy, University of

Eastern Finland, Kuopio, Finland

M. Ala-Korpela

Oulu University Hospital, Oulu, Finland

M. Ala-Korpela

Computational Medicine, School of Social and Community

Medicine and the Medical Research Council Integrative

Epidemiology Unit, University of Bristol, Bristol, UK
1,007 South Asian non-diabetic men aged 40-69 years. Follow-up was complete for 19 years in 801 European and 643 South Asian participants.

Results The serum concentrations of isoleucine, phenylalanine, tyrosine and alanine were significantly higher in South Asian men, while cross-sectional correlations of AAs with glycaemia and insulin resistance were similar in the two ethnic groups. However, most AAs were less strongly correlated with measures of obesity in the South Asian participants. Diabetes developed in 227 (35\%) South Asian and 113 (14\%) European men. Stronger adverse associations were observed between branched chain and aromatic AAs and incident

N. Sattar

Institute of Cardiovascular and Medical Sciences, University of Glasgow School of Medicine, Glasgow, UK

N. G. Forouhi

MRC Epidemiology Unit, University of Cambridge School of Clinical Medicine, Institute of Metabolic Science, Cambridge, UK

I. F. Godsland

Endocrinology and Medicine, Department of Medicine, Imperial College London, London, UK

P. M. McKeigue

Centre for Population Health Sciences, University of Edinburgh, Edinburgh, UK 
diabetes in South Asian men. Tyrosine was a particularly strong predictor of incident diabetes in South Asian individuals, even after adjustment for metabolic risk factors, including obesity and insulin resistance (adjusted OR for a $1 \mathrm{SD}$ increment, $1.47,95 \% \mathrm{CI} 1.17,1.85, p=0.001)$ compared with Europeans (OR 1.10, 0.87, 1.39, $p=0.4 ; p=0.045$ for ethnicity $\times$ tyrosine interaction).

Conclusions/interpretation Branched chain and aromatic AAs, particularly tyrosine, may be a focus for identifying novel aetiological mechanisms and potential treatment targets for diabetes in South Asian populations and may contribute to their excess risk of diabetes.

Keywords Amino acids · Cohort · Diabetes · Ethnicity . European · South Asian

$\begin{array}{ll}\text { Abbreviations } \\ \text { AA } & \text { Amino acid } \\ \text { IDI } & \text { Integrated improvement index } \\ \text { Matsuda-IR } & \text { Matsuda index of insulin resistance } \\ \text { NMR } & \text { Nuclear magnetic resonance } \\ \text { NRI } & \text { Net reclassification index } \\ \text { SABRE } & \text { Southall And Brent REvisited } \\ \text { SHR } & \text { Sub-hazard ratio }\end{array}$

\section{Introduction}

The global burden of type 2 diabetes is set to rise exponentially, with the Indian subcontinent predicted to contribute the greatest increase in the number of people with diabetes by 2030 [1]. South Asian migrant populations also experience a greater burden of diabetes than their host populations of white European origin $[2,3]$. The causal mechanisms underlying progression to type 2 diabetes remain poorly understood, and no study has yet compellingly explained the reasons for the excess risk of diabetes experienced by South Asian individuals, suggesting that complex metabolic disturbances may underlie the ethnic difference [3].

Many recent studies using metabolite profiling in European-origin populations have suggested five branched chain and aromatic amino acids (AAs) as predictors of insulin resistance and the future onset of type 2 diabetes [4-9]. A combination of three AAs predicted future diabetes in the Framingham Offspring Study, and this finding was replicated in an independent prospective cohort and in a random cohort sample [5]. More recently, a study of over 9,000 Finnish men revealed that increasing glycaemia was associated with increasing levels of six AAs (alanine, isoleucine, leucine, valine, phenylalanine and tyrosine) and with decreasing levels of histidine and glutamine; these AA associations were almost fully explained by insulin sensitivity [10].

Associations of AA profiles with insulin resistance were reported in a small cross-sectional study of 263 South Asian and Chinese men living in Singapore. The findings suggested that perturbations in AA homeostasis and increased protein turnover might underlie insulin resistance in these Chinese and South Asian men [7]. To our knowledge, there is only one other published study of AA profiles in association with insulin resistance or diabetes in South Asian individuals [11], and no studies that provide longitudinal data.

In a population-based cohort of non-diabetic British European and South Asian men followed up for 19 years, we compared by ethnicity nine circulating AAs (isoleucine, leucine, valine, phenylalanine, tyrosine, alanine, glutamine, glycine and histidine) in association with markers of insulin resistance and obesity, both in a cross-sectional analysis and as predictors of incident diabetes. Given the paucity of such studies in non-European populations, we also attempted a replication in South Asian men of the widely cited Framingham Offspring Study [5] with regard to AAs in association with incident diabetes.

\section{Methods}

The SABRE (Southall And Brent REvisited) Study involves a community-based cohort of European, South Asian and African-Caribbean origin living in North and West London. Details of the cohort have been published elsewhere [12]. Participants aged 40-69 years at baseline (1988-1991) were randomly selected from age- and sex-stratified primary care physician lists $(n=4,063)$ and workplaces $(n=795)$ in the London districts of Southall and Brent. As primary care registration is free and provides access to all health services in the UK, this forms a representative and comprehensive sampling frame. The baseline study was designed to investigate cardiometabolic risk in different ethnic groups, primarily in men. The current analyses are restricted to South Asian and European men recruited to the Southall arm of the study (19881990), due to the availability of stored baseline serum samples. We excluded those with diabetes at the baseline visit.

All the South Asian participants were migrants originating from the Indian subcontinent (52\% Punjabi Sikh, 20\% Hindu, $15 \%$ Muslim and $13 \%$ other South Asian). Interviewers recorded ethnicity on the basis of physical appearance, country of birth, name and parental origins supplemented with direct enquiry in cases of doubt. At baseline (1988-1990), the participants underwent fasting (morning) blood tests, BP measurements and anthropometry, and completed a health and lifestyle questionnaire. The questionnaire included the frequency of alcohol consumption and food frequency covering the major food groups consumed over the previous week. 
Physical activity was assessed by questionnaire, giving a summary estimate of weekly energy expenditure in daily activities plus sport, walking, cycling and strenuous activities [13]. An OGTT was also performed at the baseline visit [12]. Serum samples from participants attending the Southall study centres were stored at $-80^{\circ} \mathrm{C}$.

Deaths were flagged by the Office for National Statistics. During the period 2008-2011, survivors were invited to participate in a follow-up, including health and lifestyle questionnaires, a review of the primary care medical record and/or clinic attendance at St Mary's Hospital, London. Clinic attendees fasted overnight and underwent measurements as at baseline.

All the participants gave their written informed consent. Approval for the baseline study was obtained from Ealing, Hounslow and Spelthorne, Parkside and University College London research ethics committees, and at follow-up from the St Mary's Hospital Research Ethics Committee (ref. 07/ H0712/109).

Identifying baseline and incident diabetes The physician's diagnosis or 1999 WHO criteria [14] for fasting and OGTT blood glucose measurements defined baseline diabetes as an exclusion criterion. Incident diabetes was identified from a positive report from at least one of the following sources:

1. A review of the primary care medical record that reported a diagnosis of diabetes or the prescription of any glucoselowering medications

2. The participant questionnaire, with a recall of physiciandiagnosed diabetes together with the year of diagnosis and/or the receipt of named glucose-lowering medication

3. Clinical follow-up at 20 years with fasting or OGTT plasma glucose results meeting the 1999 WHO criteria [14]; plasma glucose was measured using hexokinase/NADP methods (Abbott Diagnostics, Maidenhead, UK)

4. A death certificate for the participant.

For the OGTT, plasma glucose and insulin were measured both at baseline and at follow-up during fasting and at $2 \mathrm{~h}$ after the oral ingestion of $75 \mathrm{~g}$ glucose [12]. Hepatic insulin resistance (HOMA-IR) was estimated using the HOMA2 calculator [15], and the Matsuda index of insulin resistance (Matsuda-IR =inverse of insulin sensitivity calculated according to the methods of Matsuda and DeFronzo $[16,17])$ provided an estimate of whole-body insulin resistance, based on the glucose and insulin values during fasting and at $2 \mathrm{~h}$ after the OGTT $[16,17]$.

Other baseline measurements Seated BP was recorded as the mean of two resting measurements. The participant's height was measured using a stadiometer. The waist and hip circumferences were measured using a fibreglass tape with a spring balance set to a constant tension of $600 \mathrm{~g}$. Harpenden calipers were used to a standard protocol to measure the skinfold thickness. Subcutaneous truncal fat was estimated by adding together the subscapular and suprailiac skinfold thicknesses.

AA quantification A high-throughput serum nuclear magnetic resonance (NMR) platform was used for AA quantification. The baseline fasting serum samples were stored at $-80^{\circ} \mathrm{C}$ and thawed overnight in a refrigerator. A proton NMR spectrum was acquired in which spectral signals from macromolecules and lipoprotein lipids were suppressed to enhance the detection of AAs. The levels of nine AAs were quantified (isoleucine, leucine, valine, phenylalanine, tyrosine, alanine, glutamine, glycine and histidine) in $\mathrm{mmol} / \mathrm{L}$. The AA profiling has previously been used in large epidemiological studies [10], and the methodology has been described in detail elsewhere [18-20].

Statistical analyses We tabulated baseline conventional risk factors and the nine measured AAs in non-diabetic participants for cross-sectional analysis and in those for whom diabetes follow-up data were available. We show medians (interquartile ranges) because of the non-normal nature of many of the distributions. Variables with a non-normal distribution were natural log-transformed and standardised to $1 \mathrm{SD}$ before further analysis. The results are presented for individual AAs, for the three-AA combination (isoleucine, phenylalanine and tyrosine) and for the five-AA combination (isoleucine, leucine, valine, phenylalanine and tyrosine) [5]. Summary variables were derived for combinations of AAs according to the formula: $z$-score of $\log X_{1}+z$-score of $\log X_{2}+z$-score of $\log$ $X_{3}$, with $X_{j}$ denoting the value of the $j$ th AA. All the scores were thereafter scaled to $1 \mathrm{SD}$.

We examined the correlations between AAs, markers of obesity and insulin resistance, and glycaemia at baseline using non-parametric methods (Spearman's rho). We tested ethnic differences in the correlations between AAs and markers of insulin resistance and obesity using Fisher's $r$ to $z$ transformation [21].

Prospective analyses used logistic regression to describe age-adjusted associations between the AAs as continuous variables and incident diabetes using ethnicity-specific models. The use of logistic regression for the primary analyses avoided the loss of cases with missing dates of diagnosis and improved the comparison with findings of the Framingham Offspring Study [5]. The associations between AAs and incident diabetes were further tested using quartiles of AA concentrations. The analyses were adjusted for age together with a group of prespecified conventional risk factors that had been identified in our earlier published analyses as important mediators of ethnic differences in the incidence of diabetes (WHR, truncal skinfold thickness, Matsuda-IR, smoking and HDLcholesterol level) [3]. We further added the self-reported 
number of units of alcohol consumption to this model as alcohol consumption has been shown to affect AA levels [22]. We assessed the interactions between ethnicity and each AA in association with incident diabetes, and examined the effects of adjustment for AAs and prespecified covariates on the ethnic differences in risk of diabetes. For comparability with the Framingham Offspring Study, model 5 was adjusted for age, fasting blood glucose and BMI.

We compared the predictive powers of AAs and conventional risk factors for risk of diabetes using the net reclassification index (NRI), the integrated improvement index (IDI) and Harrell's $C$ statistic [23, 24]. NRI cut-off points of $15 \% /$ $30 \%$ were selected a priori to reflect the high incidence rates observed as there are no thresholds for intervention with regard to diabetes risk.

In the sensitivity analyses, we repeated the above analyses of associations with incident diabetes as follows:

1. Further adjustment of model 4 for the frequency of meat/fish, dairy and green vegetable consumption and physical activity, and for serum creatinine as a marker of renal function

2. Separate analyses for the three largest South Asian subgroups (the Sikh [52\%], Hindu [20\%] and Muslim [15\%] groups)

3. The use of competing risks regression (where the competing risk was death from other causes) based on Fine and Gray's proportional subhazards methods [25].

Statistical significance was defined as $p<0.05$. We did not apply a correction for multiple testing as the associations between the AAs and glycaemic risk factors and risk of diabetes are established in populations of European origin [5, 8, 10, 26]. Statistical analyses were conducted using Stata v13 statistical software (StataCorp, College Station, TX, USA).

\section{Results}

Of 1,515 European and 1,419 South Asian male study participants from the Southall study centres, 1,423 (94\%) and 1,111 (78\%), respectively, did not have diabetes at baseline and were included in this study. Serum samples were available for 1,279 European men and 1,007 South Asian men, of whom 801 (63\%) Europeans and 643 (64\%) South Asians had known follow-up information for diabetes status (electronic supplementary material [ESM] Fig. 1).

Baseline cross-sectional analyses (Southall centre: 19881990) South Asian men were more insulin resistant and more had diagnosed hypertension compared with European men. They were more centrally obese but had lower a BMI. They were less physically active, consumed less alcohol and smoked less. They also reported a lower consumption of meat, fish and dairy products (Table 1). The serum concentrations of isoleucine, phenylalanine, tyrosine and alanine were significantly higher in South Asian men (Table 2). The findings were almost identical in those with and without information on diabetes status at follow-up. No participants were receiving lipid-lowering medications at baseline. Among the South Asian men, the concentrations of branched chain AAs and histidine were higher in Muslims than in Sikhs or Hindus, while the glutamine and glycine concentrations were higher in the Hindu than the Sikh or Muslim participants; however, the ethnic differences in AA levels compared with Europeans were maintained regardless of the South Asian subgroup.

Positive and significant correlations with markers of glycaemia and insulin resistance were observed for isoleucine, leucine, valine, phenylalanine, tyrosine and alanine in both ethnic groups. The correlations were non-significantly weaker between AAs and glycaemia and insulin resistance in South Asian men. Histidine was weakly positively correlated with measures of glycaemia and insulin resistance, and glycine and glutamine were negatively correlated in both ethnic groups. Most AAs were less strongly correlated with obesity measures in South Asian men (significantly so for branched chain AAs and alanine) (Table 3).

Prospective analyses: AAs and incident diabetes (19882011) The median duration of follow-up was 19 years. Diabetes developed in 227 South Asian men (35\%) and $113 \mathrm{Eu}-$ ropean men (14\%). The median (interquartile range) number of years from baseline to the development of diabetes was 15 $(11,18)$ in Europeans and $14(9,18)$ in South Asians $(p=$ 0.075 ), and the median (interquartile range) age at diagnosis of diabetes was $67(60,71)$ years in the European participants and $62(57,68)$ years in the South Asian participants $(p<0.0002)$.

In logistic regression analyses adjusted only for age, all AAs and AA combinations were associated with incident diabetes in both ethnic groups (Table 4, model 1), with the exception of phenylalanine, glutamine and histidine in European men and glycine in South Asian men. Similar results were obtained when the AAs were analysed in quartiles (see ESM Table 1). Multivariable analyses for the European men showed that, with the exception of glycine and isoleucine, all linear associations (per SD log-transformed AA level) were markedly attenuated following adjustments for obesity and further attenuated on adjustment for insulin resistance and other conventional risk factors (Table 4, models 2-5). Positive associations between incident diabetes and individual AAs and the three- and five-AA combinations were generally more marked in South Asian individuals and only partially attenuated on adjustment for obesity measures and fasting glucose levels (Table 4, models 2 and 5). 
Table 1 Baseline characteristics of European and South Asian men without baseline diabetes

\begin{tabular}{|c|c|c|c|c|c|c|}
\hline \multirow[t]{2}{*}{ Baseline characteristics } & \multicolumn{3}{|c|}{$\begin{array}{l}\text { All with stored baseline serum (included } \\
\text { in cross-sectional analyses) }\end{array}$} & \multicolumn{3}{|c|}{$\begin{array}{l}\text { All with stored baseline serum and data } \\
\text { regarding diabetes status at follow-up } \\
\text { (included in prospective analyses) }\end{array}$} \\
\hline & European & South Asian & $\begin{array}{l}p \text { values } \\
\text { for ethnic } \\
\text { difference }\end{array}$ & European & South Asian & $\begin{array}{l}p \text { values } \\
\text { for ethnic } \\
\text { difference }\end{array}$ \\
\hline Number of individuals & 1,279 & 1,007 & & 801 & 643 & \\
\hline Age, mean \pm SD & $52.9 \pm 7.3$ & $50.6 \pm 7.0$ & $<0.001$ & $52.6 \pm 7.2$ & $50.6 \pm 7.0$ & $<0.001$ \\
\hline Fasting glucose $(\mathrm{mmol} / \mathrm{l})$ & $5.36(5.04,5.69)$ & $5.46(5.09,5.83)$ & $<0.001$ & $5.36(5.03,5.71)$ & $5.45(5.06,5.82)$ & $<0.001$ \\
\hline $2 \mathrm{~h}$ glucose $(\mathrm{mmol} / \mathrm{l})$ & $4.85(4.04,5.66)$ & $5.34(4.50,6.20)$ & $<0.001$ & $4.86(4.04,5.72)$ & $5.27(4.45,6.08)$ & $<0.001$ \\
\hline Fasting insulin (pmol/1) & $50.00(33.34,72.23)$ & $69.80(47.92,98.62)$ & $<0.001$ & $51.39(34.03,73.62)$ & $69.10(47.92,98.62)$ & $<0.001$ \\
\hline $2 \mathrm{~h}$ insulin (pmol/l) & $\begin{array}{l}134.04(79.87 \\
234.05)\end{array}$ & $\begin{array}{l}274.67(158.35 \\
504.90)\end{array}$ & $<0.001$ & $\begin{array}{l}141.68(33.34 \\
235.44)\end{array}$ & $\begin{array}{l}275.37(159.74 \\
497.26)\end{array}$ & $<0.001$ \\
\hline HDL-cholesterol (mmol/l) & $\begin{array}{l}n=1,272 \\
1.24(1.05,1.48)\end{array}$ & $\begin{array}{l}n=1,000 \\
1.15(0.98,1.35)\end{array}$ & $<0.001$ & $\begin{array}{l}n=795 \\
1.24(1.06,1.48)\end{array}$ & $\begin{array}{l}n=640 \\
1.15(0.99,1.37)\end{array}$ & $<0.001$ \\
\hline Triacylglycerols (mmol/l) & $1.43(1.02,2.09)$ & $1.71(1.15,2.51)$ & $<0.001$ & $1.36(0.99,2.0)$ & $1.60(1.09,2.31)$ & $<0.001$ \\
\hline Serum creatinine $(\mu \mathrm{mol} / \mathrm{l})$ & $60.37(54.60,67.24)$ & $57.47(51.43,64.03)$ & $<0.001$ & $60.79(55.0,67.79)$ & $57.47(51.78,63.15)$ & $<0.001$ \\
\hline HOMA-IR & $0.8(0.5,1.2)$ & $1.1(0.8,1.6)$ & $<0.001$ & $0.8(0.6,1.2)$ & $1.1(0.8,1.6)$ & $<0.001$ \\
\hline Matsuda-IR & $0.20(0.13,0.32)$ & $0.35(0.22,0.58)$ & $<0.001$ & $0.21(0.13,0.33)$ & $0.35(0.22,0.57)$ & $<0.001$ \\
\hline Waist circumference $(\mathrm{cm})$ & $90.9(84.5,97.7)$ & $92.1(86.1,98.5)$ & 0.02 & $90.6(84.7,97.5)$ & $91.5(85.7,97.6)$ & 0.06 \\
\hline WHR & $0.94(0.90,0.98)$ & $0.98(0.93,1.02)$ & $<0.001$ & $0.94(0.90,0.98)$ & $0.98(0.93,1.02)$ & $<0.001$ \\
\hline Truncal skinfold thickness $(\mathrm{cm})$ & $3.7(2.9,4.7)$ & $4.5(3.7,5.5)$ & $<0.001$ & $3.7(2.9,4.7)$ & $4.5(3.7,5.7)$ & $<0.001$ \\
\hline BMI $\left(\mathrm{kg} / \mathrm{m}^{2}\right)$ & $25.65(23.68,27.96)$ & $25.46(23.50,27.53)$ & 0.03 & $25.63(23.69,27.78)$ & $25.28(23.30,27.23)$ & 0.05 \\
\hline Systolic BP (mmHg) & $121(111,132)$ & $122(112,133)$ & 0.04 & $119(110,132)$ & $121(112,133)$ & 0.02 \\
\hline Treated hypertension & $103(8)$ & $117(12)$ & 0.004 & $63(8)$ & $81(13)$ & 0.003 \\
\hline Smoking, never/ex/current (\%) & $26 / 41 / 33$ & $74 / 9 / 16$ & $<0.001$ & $28 / 40 / 32$ & $74 / 10 / 16$ & $<0.001$ \\
\hline Alcohol (units per week) & $\begin{array}{l}n=1,244 \\
11(3,23)\end{array}$ & $\begin{array}{l}n=969 \\
3(0,15)\end{array}$ & $<0.001$ & $\begin{array}{l}n=774 \\
12(3,24)\end{array}$ & $\begin{array}{l}n=619 \\
3(0,14)\end{array}$ & $<0.001$ \\
\hline $\begin{array}{l}\text { Meat and fish consumption during } \\
\text { previous } 7 \text { days, quartiles of } \\
\text { frequency, lowest to highest (\%) }\end{array}$ & $4 / 15 / 40 / 41$ & $35 / 26 / 25 / 13$ & $<0.0001$ & $3 / 14 / 40 / 42$ & $36 / 26 / 24 / 15$ & $<0.0001$ \\
\hline $\begin{array}{l}\text { Dairy product consumption during } \\
\text { previous } 7 \text { days, none } / 1 \\
\text { day } / 2-3 \text { days } / \text { most days }(\%)\end{array}$ & $2 / 4 / 11 / 83$ & $7 / 8 / 19 / 66$ & $<0.0001$ & $2 / 4 / 11 / 83$ & $6 / 6 / 20 / 67$ & $<0.0001$ \\
\hline $\begin{array}{l}\text { Green vegetable consumption during } \\
\text { previous } 7 \text { days, none } / 1 \\
\text { day/2-3 days/most days }(\%)\end{array}$ & $7 / 7 / 32 / 54$ & $4 / 8 / 35 / 53$ & 0.017 & $6 / 7 / 31 / 56$ & $4 / 8 / 36 / 51$ & 0.057 \\
\hline Physical activity $\left(\mathrm{kJ} \times 10^{3} /\right.$ week $)$ & $11(7,16)$ & $9(5,13)$ & $<0.001$ & $11(7,16)$ & $9(5,13)$ & $<0.001$ \\
\hline Education (years) & $10(9,11)$ & $12(10,15)$ & $<0.001$ & $10(9,11)$ & $12(10,15)$ & $<0.001$ \\
\hline
\end{tabular}

Data are median (interquartile range), $n(\%)$ unless otherwise stated

Additional adjustment for Matsuda-IR, smoking and HDLcholesterol level and then alcohol consumption resulted in further attenuation for branched chain AAs, phenylalanine, alanine, glutamine and histidine (models 3 and 4). However, the association for tyrosine was particularly marked among the South Asian participants even after full adjustment (fully adjusted OR for a 1 SD increment in the log-transformed level: 1.47 [1.17, 1.85] vs Europeans: 1.10 [0.87, 1.39]; ethnicity $\times$ tyrosine interaction $p=0.045$ (fully adjusted)). Similarly, the three- and five-AA combinations of isoleucine, leucine, valine, phenylalanine and tyrosine were also more strongly associated with incident diabetes in South Asian participants (fully adjusted ORs [model 4]: South Asians: 1.32 [1.04, 1.68] and 1.31[1.04, 1.66] vs European participants: $1.03[0.82,1.29]$ and $1.05[0.84,1.30])$, with weak evidence of ethnicity interactions ( $p=0.10$ and 0.13 , respectively) (Table 4, model 4). Glycine was negatively associated with incident diabetes in European but not in South Asian men (interaction $p=0.06$ ).

Comparisons with the Framingham Offspring Study are shown following adjustment for age, fasting glucose level and BMI (Table 4, model 5). In European men, the model 5 
Table 2 Baseline AA levels of European and South Asian men (all without baseline diabetes)

\begin{tabular}{|c|c|c|c|c|c|c|}
\hline \multirow{2}{*}{$\begin{array}{l}\text { Amino acids }(\mathrm{mmol} / \mathrm{l}) \\
\text { Baseline characteristics }\end{array}$} & \multicolumn{3}{|c|}{$\begin{array}{l}\text { All with stored baseline serum (included in } \\
\text { cross-sectional analyses) }\end{array}$} & \multicolumn{3}{|c|}{$\begin{array}{l}\text { All with stored baseline serum and data regarding } \\
\text { diabetes status at follow-up (included in prospective } \\
\text { analyses) }\end{array}$} \\
\hline & European & South Asian & $\begin{array}{l}p \text { values } \\
\text { for ethnic } \\
\text { difference }\end{array}$ & European & South Asian & $\begin{array}{l}p \text { values } \\
\text { for ethnic } \\
\text { difference }\end{array}$ \\
\hline Number & 1,279 & 1,007 & & 801 & 643 & \\
\hline Isoleucine & $0.057(0.050,0.066)$ & $0.060(0.052,0.068)$ & $<0.0001$ & $0.058(0.050,0.066)$ & $0.060(0.052,0.068)$ & 0.002 \\
\hline Leucine & $0.091(0.080,0.11)$ & $0.093(0.083,0.11)$ & 0.17 & $0.092(0.081,0.11)$ & $0.092(0.083,0.10)$ & 0.8 \\
\hline Valine & $0.179(0.158,0.202)$ & $0.178(0.156,0.199)$ & 0.17 & $0.180(0.158,0.204)$ & $0.176(0.155,0.198)$ & 0.017 \\
\hline Phenylalanine & $0.092(0.084,0.10)$ & $0.094(0.086,0.10)$ & 0.019 & $0.092(0.084,0.10)$ & $0.094(0.085,0.10)$ & 0.12 \\
\hline Tyrosine & $0.054(0.048,0.061)$ & $0.060(0.053,0.067)$ & $<0.0001$ & $0.054(0.048,0.061)$ & $0.059(0.052,0.067)$ & $<0.0001$ \\
\hline $\begin{array}{l}\text { Isoleucine, phenylalanine, } \\
\text { tyrosine combined }\end{array}$ & $0.205(0.186,0.2228)$ & $0.213(0.197,0.234)$ & $<0.0001$ & $0.205(0.186,0.228)$ & $0.213(0.197,0.233)$ & $<0.0001$ \\
\hline Alanine & $0.326(0.290,0.365)$ & $0.335(0.301,0.375)$ & $<0.0001$ & $0.328(0.290,0.367)$ & $0.335(0.302,0.374)$ & 0.001 \\
\hline Glutamine & $0.384(0.257,0.448)$ & $0.410(0.309,0.471)$ & $<0.0001$ & $0.385(0.262,0.450)$ & $0.415(0.317,0.473)$ & $<0.0001$ \\
\hline Glycine & $\begin{array}{l}n=1,267 \\
0.288(0.262,0.318)\end{array}$ & $\begin{array}{l}n=998 \\
0.282(0.256,0.311)\end{array}$ & 0.006 & $\begin{array}{l}n=794 \\
0.287(0.262,0.316)\end{array}$ & $\begin{array}{l}n=638 \\
0.282(0.257,0.312)\end{array}$ & 0.10 \\
\hline Histidine & $0.077(0.069,0.088)$ & $0.078(0.070,0.089)$ & 0.3 & $0.077(0.068,0.088)$ & $0.078(0.070,0.090)$ & 0.14 \\
\hline
\end{tabular}

Data are median (interquartile range)

Table 3 Correlations between AAs and markers of glycaemia/insulin resistance and obesity at baseline in non-diabetic men

\begin{tabular}{|c|c|c|c|c|c|c|c|c|c|c|c|}
\hline Spearman's $\rho^{\mathrm{a}}$ & $\begin{array}{l}\text { European men } \\
(n=1,279) \\
\text { South Asian } \\
\text { men }(n=1,007)\end{array}$ & $\begin{array}{l}\text { Fasting } \\
\text { glucose }\end{array}$ & $\begin{array}{l}2 \mathrm{~h} \\
\text { glucose }\end{array}$ & $\begin{array}{l}\text { Fasting } \\
\text { insulin }\end{array}$ & $\begin{array}{l}2 \mathrm{~h} \\
\text { insulin }\end{array}$ & HOMA-IR & Matsuda-IR & WHR & $\begin{array}{l}\text { Waist } \\
\text { circumference }\end{array}$ & BMI & $\begin{array}{l}\text { Truncal skinfold } \\
\text { thickness }\end{array}$ \\
\hline \multirow[t]{2}{*}{ Isoleucine } & European men & 0.16 & 0.12 & 0.35 & 0.28 & 0.35 & 0.34 & 0.30 & 0.34 & 0.31 & 0.32 \\
\hline & South Asian men & 0.13 & 0.13 & 0.34 & 0.32 & 0.34 & 0.36 & $0.21^{\mathrm{b}}$ & $0.25^{\mathrm{b}}$ & $0.23^{\mathrm{b}}$ & 0.30 \\
\hline \multirow[t]{2}{*}{ Leucine } & European men & 0.18 & 0.10 & 0.30 & 0.20 & 0.31 & 0.27 & 0.23 & 0.27 & 0.27 & 0.29 \\
\hline & South Asian men & 0.14 & 0.07 & 0.26 & 0.21 & 0.26 & 0.25 & $0.14^{\mathrm{b}}$ & $0.19^{\mathrm{b}}$ & $0.18^{\mathrm{b}}$ & 0.25 \\
\hline \multirow[t]{2}{*}{ Valine } & European men & 0.18 & 0.14 & 0.32 & 0.23 & 0.33 & 0.30 & 0.23 & 0.28 & 0.29 & 0.31 \\
\hline & South Asian men & 0.15 & 0.12 & 0.26 & 0.27 & 0.27 & 0.29 & $0.13^{\mathrm{b}}$ & $0.19^{\mathrm{b}}$ & 0.22 & 0.29 \\
\hline \multirow[t]{2}{*}{ Phenylalanine } & European men & 0.19 & 0.13 & 0.24 & 0.18 & 0.25 & 0.23 & 0.26 & 0.25 & 0.22 & 0.21 \\
\hline & South Asian men & 0.09 & 0.11 & 0.21 & 0.18 & 0.21 & 0.22 & 0.22 & 0.22 & 0.15 & 0.20 \\
\hline \multirow[t]{2}{*}{ Tyrosine } & European men & 0.22 & 0.11 & 0.36 & 0.26 & 0.36 & 0.33 & 0.32 & 0.35 & 0.34 & 0.31 \\
\hline & South Asian men & 0.23 & 0.14 & 0.29 & 0.24 & 0.30 & 0.30 & 0.30 & 0.35 & 0.30 & 0.27 \\
\hline \multirow[t]{2}{*}{ Alanine } & European men & 0.27 & 0.12 & 0.34 & 0.23 & 0.35 & 0.31 & 0.20 & 0.24 & 0.23 & 0.22 \\
\hline & South Asian men & 0.24 & 0.15 & 0.27 & 0.20 & 0.29 & 0.27 & 0.17 & $0.16^{\mathrm{b}}$ & 0.17 & 0.23 \\
\hline \multirow[t]{2}{*}{ Glutamine } & European men & -0.11 & -0.09 & -0.18 & -0.07 & -0.18 & -0.14 & -0.11 & -0.12 & -0.12 & -0.16 \\
\hline & South Asian men & -0.07 & $-0.03^{\mathrm{a}}$ & -0.17 & $-0.04^{\mathrm{a}}$ & -0.17 & -0.10 & -0.08 & -0.08 & -0.07 & -0.15 \\
\hline \multirow[t]{2}{*}{ Glycine } & $\begin{array}{c}\text { European men } \\
\quad(n=1,267)\end{array}$ & $0.04^{\mathrm{a}}$ & $-0.0^{\mathrm{a}}$ & -0.07 & -0.10 & -0.05 & -0.09 & -0.09 & -0.10 & -0.11 & -0.05 \\
\hline & $\begin{array}{l}\text { South Asian men } \\
\quad(n=998)\end{array}$ & $0.03^{\mathrm{a}}$ & $0.003^{\mathrm{a}}$ & $-0.06^{\mathrm{a}}$ & -0.09 & $-0.06^{\mathrm{a}}$ & -0.08 & -0.08 & -0.11 & -0.15 & $-0.01^{\mathrm{a}}$ \\
\hline \multirow[t]{2}{*}{ Histidine } & European men & 0.08 & 0.08 & 0.11 & $0.04^{\mathrm{a}}$ & 0.10 & 0.09 & $0.03^{\mathrm{a}}$ & 0.07 & 0.07 & 0.14 \\
\hline & South Asian men & $0.07^{\mathrm{a}}$ & $0.03^{\mathrm{a}}$ & $0.04^{\mathrm{a}}$ & $0.01^{\mathrm{a}}$ & $0.04^{\mathrm{a}}$ & $0.03^{\mathrm{a}}$ & $0.01^{\mathrm{a}}$ & $0.02^{\mathrm{a}}$ & $0.02^{\mathrm{a}}$ & 0.16 \\
\hline
\end{tabular}

${ }^{a} p$ values for correlations within ethnic group were $<0.05$, unless marked ${ }^{\text {a }}$

${ }^{\mathrm{b}} p$ values for ethnic group difference in correlation coefficient $<0.05$ 
Table 4 Associations between AAs and incident diabetes

\begin{tabular}{|c|c|c|c|c|c|c|}
\hline $\begin{array}{l}\text { OR per SD log-transformed } \\
\text { AA concentration }\end{array}$ & & European men & $p$ values & South Asian men & $p$ values & $\begin{array}{l}p \text { values for interaction } \\
\text { ethnicity } \times \mathrm{AA}\end{array}$ \\
\hline \multirow[t]{5}{*}{ Isoleucine } & Model 1 & $1.48(1.20,1.83)$ & $3 \times 10^{-4}$ & $1.58(1.30,1.93)$ & $5 \times 10^{-6}$ & 0.7 \\
\hline & Model 2 & $1.27(1.02,1.58)$ & 0.032 & $1.34(1.10,1.65)$ & 0.004 & 0.6 \\
\hline & Model 3 & $1.09(0.94,1.00)$ & 0.5 & $1.22(0.98,1.51)$ & 0.069 & 0.7 \\
\hline & Model 4 & $1.09(0.87,1.38)$ & 0.4 & $1.19(0.95,1.48)$ & 0.13 & 0.6 \\
\hline & Model 5 & $1.21(0.97,1.51)$ & 0.089 & $1.42(1.16,1.75)$ & 0.001 & 0.3 \\
\hline \multirow[t]{5}{*}{ Leucine } & Model 1 & $1.30(1.10,1.55)$ & 0.003 & $1.50(1.23,1.82)$ & $6 \times 10^{-5}$ & 0.3 \\
\hline & Model 2 & $1.19(0.99,1.43)$ & 0.071 & $1.34(1.10,1.65)$ & 0.004 & 0.4 \\
\hline & Model 3 & $1.09(0.88,1.34)$ & 0.4 & $1.31(1.06,1.62)$ & 0.014 & 0.2 \\
\hline & Model 4 & $1.10(0.89,1.35)$ & 0.4 & $1.22(0.98,1.52)$ & 0.074 & 0.3 \\
\hline & Model 5 & $1.11(0.92,1.35)$ & 0.3 & $1.36(1.11,1.68)$ & 0.004 & 0.17 \\
\hline \multirow[t]{5}{*}{ Valine } & Model 1 & $1.29(1.06,1.58)$ & 0.013 & $1.53(1.26,1.84)$ & $1 \times 10^{-5}$ & 0.2 \\
\hline & Model 2 & $1.12(0.90,1.38)$ & 0.3 & $1.35(1.11,1.64)$ & 0.003 & 0.2 \\
\hline & Model 3 & $1.02(0.81,1.28)$ & 0.9 & $1.24(1.01,1.54)$ & 0.044 & 0.3 \\
\hline & Model 4 & $1.00(0.80,1.27)$ & 0.9 & $1.24(1.00,1.54)$ & 0.052 & 0.3 \\
\hline & Model 5 & $1.04(0.84,1.29)$ & 0.7 & $1.32(1.08,1.62)$ & 0.007 & 0.14 \\
\hline \multirow[t]{5}{*}{ Phenylalanine } & Model 1 & $1.13(0.93,1.37)$ & 0.2 & $1.36(1.12,1.64)$ & 0.002 & 0.19 \\
\hline & Model 2 & $1.00(0.82,1.22)$ & 1.0 & $1.19(0.98,1.45)$ & 0.076 & 0.2 \\
\hline & Model 3 & $0.91(0.74,1.11)$ & 0.4 & $1.12(0.91,1.37)$ & 0.3 & 0.2 \\
\hline & Model 4 & $0.91(0.74,1.12)$ & 0.4 & $1.08(0.88,1.33)$ & 0.5 & 0.2 \\
\hline & Model 5 & $0.97(0.80,1.18)$ & 0.8 & $1.29(1.06,1.57)$ & 0.011 & 0.054 \\
\hline \multirow[t]{5}{*}{ Tyrosine } & Model 1 & $1.44(1.17,1.76)$ & $5 \times 10^{-4}$ & $1.92(1.57,2.35)$ & $3 \times 10^{-10}$ & 0.049 \\
\hline & Model 2 & $1.22(0.98,1.51)$ & 0.071 & $1.66(1.33,2.03)$ & $5 \times 10^{-6}$ & 0.053 \\
\hline & Model 3 & $1.07(0.85,1.35)$ & 0.6 & $1.55(1.24,1.93)$ & $1 \times 10^{-4}$ & 0.038 \\
\hline & Model 4 & $1.10(0.87,1.39)$ & 0.4 & $1.47(1.17,1.85)$ & 0.001 & 0.045 \\
\hline & Model 5 & $1.11(0.90,1.39)$ & 0.3 & $1.56(1.26,1.93)$ & $5 \times 10^{-5}$ & 0.047 \\
\hline \multirow[t]{5}{*}{ Alanine } & Model 1 & $1.27(1.03,1.55)$ & 0.022 & $1.45(1.20,1.75)$ & $1 \times 10^{-4}$ & 0.3 \\
\hline & Model 2 & $1.13(0.91,1.39)$ & 0.3 & $1.31(1.08,1.59)$ & 0.005 & 0.3 \\
\hline & Model 3 & $1.06(0.85,1.33)$ & 0.6 & $1.21(0.99,1.49)$ & 0.057 & 0.5 \\
\hline & Model 4 & $1.12(0.89,1.40)$ & 0.3 & $1.15(0.94,1.42)$ & 0.17 & 0.8 \\
\hline & Model 5 & $1.03(0.84,1.28)$ & 0.8 & $1.25(1.03,1.51)$ & 0.025 & 0.2 \\
\hline \multirow[t]{5}{*}{ Glutamine } & Model 1 & $0.91(0.76,1.09)$ & 0.3 & $0.82(0.70,0.97)$ & 0.022 & 0.4 \\
\hline & Model 2 & $0.96(0.79,1.16)$ & 0.6 & $0.86(0.73,1.03)$ & 0.10 & 0.4 \\
\hline & Model 3 & $0.98(0.80,1.19)$ & 0.8 & $0.85(0.71,1.03)$ & 0.091 & 0.2 \\
\hline & Model 4 & $0.94(0.77,1.15)$ & 0.5 & $0.94(0.77,1.14)$ & 0.5 & 0.4 \\
\hline & Model 5 & $0.94(0.77,1.15)$ & 0.6 & $0.88(0.73,1.05)$ & 0.15 & 0.6 \\
\hline \multirow[t]{5}{*}{ Glycine } & Model 1 & $0.77(0.63,0.93)$ & 0.007 & $0.95(0.80,1.12)$ & 0.5 & 0.10 \\
\hline & Model 2 & $0.78(0.64,0.96)$ & 0.016 & $0.98(0.82,1.17)$ & 0.8 & 0.11 \\
\hline & Model 3 & $0.75(0.61,0.93)$ & 0.010 & $0.99(0.82,1.20)$ & 0.9 & 0.047 \\
\hline & Model 4 & $0.76(0.61,0.94)$ & 0.011 & $0.98(0.81,1.19)$ & 0.8 & 0.058 \\
\hline & Model 5 & $0.78(0.64,0.96)$ & 0.019 & $0.96(0.80,1.15)$ & 0.7 & 0.13 \\
\hline \multirow[t]{5}{*}{ Histidine } & Model 1 & $0.98(0.82,1.18)$ & 0.8 & $1.19(0.99,1.43)$ & 0.062 & 0.15 \\
\hline & Model 2 & $0.94(0.78,1.15)$ & 0.6 & $1.16(0.96,1.41)$ & 0.13 & 0.15 \\
\hline & Model 3 & $0.93(0.75,1.15)$ & 0.5 & $1.20(0.98,1.46)$ & 0.084 & 0.077 \\
\hline & Model 4 & $0.93(0.75,1.15)$ & 0.6 & $1.15(0.93,1.42)$ & 0.2 & 0.10 \\
\hline & Model 5 & $0.92(0.75,1.12)$ & 0.4 & $1.13(0.93,1.38)$ & 0.2 & 0.14 \\
\hline \multirow[t]{3}{*}{ Isoleucine, phenylalanine, tyrosine } & Model 1 & $1.39(1.14,1.69)$ & 0.001 & $1.85(1.50,2.29)$ & $1 \times 10^{-8}$ & 0.051 \\
\hline & Model 2 & $1.18(0.95,1.46)$ & 0.13 & $1.54(1.23,1.92)$ & $1 \times 10^{-4}$ & 0.083 \\
\hline & Model 3 & $1.02(0.81,1.27)$ & 0.9 & $1.39(1.10,1.75)$ & 0.001 & 0.096 \\
\hline
\end{tabular}


Table 4 (continued)

\begin{tabular}{lllllll}
\hline $\begin{array}{l}\text { OR per SD log-transformed } \\
\text { AA concentration }\end{array}$ & & European men & $p$ values & South Asian men & $\begin{array}{c}p \text { values } \\
p \text { values for interaction } \\
\text { ethnicity } \times \text { AA }\end{array}$ \\
\hline & Model 4 & $1.03(0.82,1.29)$ & 0.8 & $1.32(1.04,1.68)$ & 0.022 & 0.10 \\
& Model 5 & $1.10(0.89,1.36)$ & 0.4 & $1.57(1.26,1.96)$ & $5 \times 10^{-5}$ & 0.029 \\
Branched chain + aromatic AAs & Model 1 & $1.36(1.13,1.65)$ & 0.001 & $1.79(1.45,2.20)$ & $5 \times 10^{-8}$ & 0.061 \\
& Model 2 & $1.18(0.96,1.45)$ & 0.11 & $1.51(1.21,1.87)$ & $1 \times 10^{-5}$ & 0.10 \\
& Model 3 & $1.04(0.83,1.29)$ & 0.8 & $1.38(1.10,1.73)$ & 0.006 & 0.12 \\
& Model 4 & $1.05(0.84,1.30)$ & 0.7 & $1.31(1.04,1.66)$ & 0.023 & 0.13 \\
& Model 5 & $1.10(0.89,1.35)$ & 0.4 & $1.52(1.22,1.89)$ & $2 \times 10^{-4}$ & 0.040 \\
\hline
\end{tabular}

Model 1: adjusted for age

Model 2: adjusted for age, WHR and truncal skinfold thickness

Model 3: adjusted for age, WHR, truncal skinfold thickness, Matsuda-IR, HDL-cholesterol level and current smoking

Model 4: model 3 plus alcohol consumption

Model 5: adjusted for age, fasting glucose level and BMI (for comparison with the Framingham Offspring Study [5])

adjusted ORs per $1 \mathrm{SD}$ increment in the log-transformed three-AA (isoleucine, phenylalanine and tyrosine) and fiveAA (isoleucine, leucine, valine, phenylalanine and tyrosine) combinations were virtually identical and weak: $1.10(0.89$, $1.36)$ and $1.10(0.89,1.35)$. In contrast, the ORs were greater in South Asian men $(1.57$ [1.26, 1.96] and 1.52 [1.22, 1.89], respectively; Table 4, model 5). In South Asian men, the ORs for the upper vs lowest quartile were $3.11(1.71,5.67)$ and $2.55(1.46,4.48)$ for the three- and five-AA combinations, respectively, with evidence of linear associations. The corresponding categorical associations for Europeans were weaker (ORs for the upper vs lower quartile $1.99[1.07,3.67]$ and 1.98 [1.05, 3.76], respectively) (ESM Table 1, model 5).

In aiming to predict incident diabetes, no improvements in the $\mathrm{C}$ statistic, NRI or IDI were observed in European men on the addition of tyrosine or either the three- or five-AA combination to models containing prespecified conventional risk factors. However, in South Asian men, the IDI and NRI significantly improved with the addition of tyrosine, although only discrimination (IDI) significantly improved with the addition of the three- and five-AA combinations (ESM Table 2).

Prospective analyses: AAs and ethnic differences in incidence of diabetes In age-adjusted models, South Asian men had a 3.18 -fold (95\% CI 2.46, 4.12, $\left.p=1.5 \times 10^{-18}\right)$ greater risk of developing diabetes than European men (Fig. 1). Additional adjustment for baseline tyrosine level reduced the ethnic difference in risk of diabetes to the greatest extent (OR 2.64 $[2.02,3.44] ; 17 \%$ reduction). Adjustment for the prespecified combination of conventional risk factors reduced the ethnic OR to $2.10(1.56,2.82)$. Further adjustment for AAs modestly altered the ethnic difference in risk of diabetes; the addition of tyrosine to the full model led to the largest reduction in OR, to $1.99(1.48,2.69 ; 5 \%$ reduction) (Fig. 1).
Sensitivity analyses Sensitivity analyses that included adjustment for diet, physical activity or serum creatinine, or excluded participants with impaired fasting glucose or impaired glucose tolerance, produced similar results to those reported above. There were no significant age-adjusted interactions between the main South Asian subgroups (Punjabi Sikh, Hin$\mathrm{du}$ and Muslim) and AAs in association with incident diabetes (all interaction $p$ values $>0.10$ ).

Prospective analyses using competing risks regression resulted in the loss of 23 cases of incident diabetes without dates of diagnosis, but demonstrated similar ethnic differences in the incidence of diabetes (age-adjusted subhazard ratio [SHR] for South Asian vs European participants 2.90 [2.33, 3.61]), which were again most markedly attenuated on the addition of baseline tyrosine (SHR 2.52 [2.00, 3.20]). Competing risks regression also demonstrated similar associations between AAs and incident diabetes, for example the ageadjusted SHR per SD log-transformed tyrosine in Europeans of $1.31(1.05,1.64)$ and in South Asians of $1.65(1.44,1.89)$.

\section{Discussion}

We report novel ethnicity-specific data regarding the associations between AAs and incident diabetes in over 19 years of follow-up. We demonstrate significant positive associations between baseline branched chain AAs, tyrosine and alanine and incident diabetes in middle-aged European men in accord with previous studies [4-9, 27]. In South Asian men, the same AAs, plus phenylalanine, were more strongly associated with incident diabetes compared with Europeans. In European men, obesity and insulin resistance, together with age, smoking, HDL-cholesterol level and alcohol consumption partially or completely accounted for the majority of 
Fig. 1 ORs for ethnic differences in the incidence of diabetes in South Asian vs European men, adjusted for age and AAs, and for conventional risk factors and AAs. ${ }^{a}$ Adjusted for age plus each $\mathrm{AA}$ or combination in turn. ${ }^{\mathrm{b}}$ Adjusted for age, WHR, truncal skinfold thickness, Matsuda-IR, HDL-cholesterol (HDL-C) level and current smoking, plus each AA or combination in turn

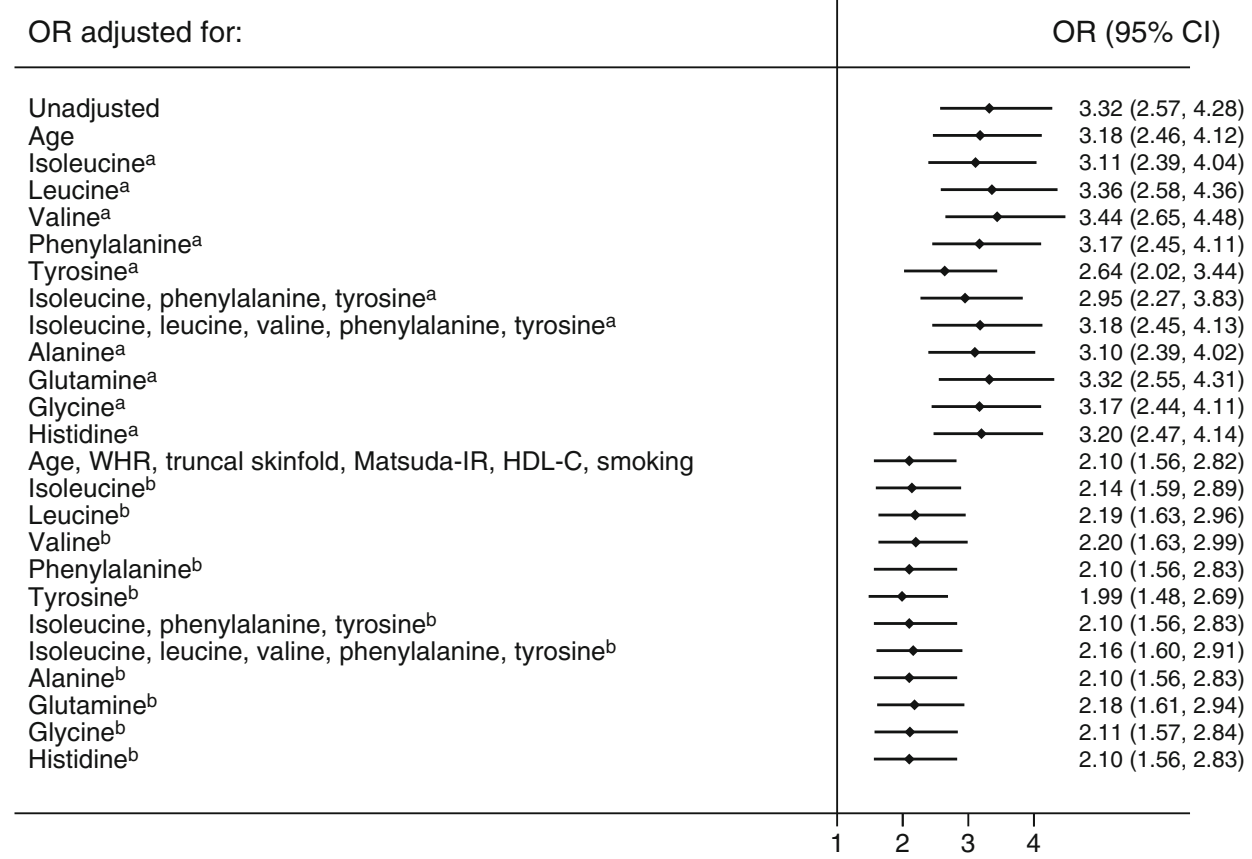

associations between AAs incident diabetes. In contrast, in South Asian men, the adverse associations were, with the exception of phenylalanine, generally only moderately attenuated on adjustment for insulin resistance, smoking, HDLcholesterol, alcohol intake and creatinine level, and remained statistically significant. Tyrosine and the combination of branched chain and aromatic AAs were particularly strongly associated with incident diabetes even after multivariable adjustment in South Asian men. Adjustment for tyrosine made a small (approximately 5\%) contribution to explaining the ethnic difference in the incidence of diabetes.

Cross-sectional associations at baseline between AAs and markers of insulin resistance and glycaemia generally followed the patterns observed for incident diabetes, with the exception of histidine, which was minimally associated with markers of insulin resistance in South Asian men. Associations between branched chain AAs and obesity, although present, were weaker in South Asian than European participants. The latter findings, together with the weaker attenuation of AA associations with incident diabetes, suggest that conventional measures may not capture the best indicators of diabetes risk in South Asian individuals.

Comparison with other studies Although it did not have European comparators and was only a small cross-sectional analysis, the study by Tai et al [7] reported strong associations in South Asian and Chinese men of relatively low body weight between insulin resistance and branched chain and aromatic AAs and for a combination of isoleucine, leucine, phenylalanine, tyrosine and methionine. These associations were independent of dietary protein intake and BMI [7]. This is compatible with our cross-sectional finding of weaker associations between obesity and branched chain AAs in South Asian men, suggesting that increases in these AA levels may be driven by insulin resistance to a greater extent than obesity in South Asian individuals or that measures of obesity may be less satisfactory in this population.

Another small cross-sectional study in younger (age 3545 years) Indian Asians living in India found strong correlations between isoleucine, leucine and phenylalanine and obesity in people with a high BMI both with and without type 2 diabetes, and lower correlations in those with a low BMI and no diabetes [11]. There was no comparator group, so we cannot compare the relative strength of the correlations. However, these findings are not inconsistent with ours, in that we also found significant correlations between branched chain and aromatic amino acids and measures of obesity, albeit weaker in the South Asian than in the European participants.

The findings of the Framingham Offspring Study for three AAs and for five AAs in the lower risk random cohort sample adjusted for age, fasting glucose level and BMI are comparable with our findings for European men for the upper vs lower quartiles of AA levels (although the linear association is attenuated), but they somewhat understate the associations observed in the higher risk South Asian men in our study [5]. We had anticipated that the findings in the European and South Asian participants in the SABRE Study would most closely resemble the Framingham random cohort. European SABRE participants had comparable baseline levels of glucose, insulin and obesity, but were younger, were less likely to be hypertensive and had lower triacylglycerol levels, perhaps explaining the somewhat weaker associations of AAs with 
incident diabetes compared with those observed in the Framingham random cohort. South Asian SABRE participants, who were also younger, were more centrally obese and had higher fasting glucose and insulin levels, but experienced similar triacylglycerol levels and less hypertension than the Framingham random cohort. Associations between AAs and incident diabetes in South Asian individuals more closely resembled the stronger associations seen in the Framingham replication cohort (Malmo Diet and Cancer cohort [28]) and Framingham high-risk propensity matched cases and controls, despite having markedly lower overall obesity (BMI) than the latter group [5].

Possible mechanisms Branched chain and aromatic AAs have been associated with insulin-resistant states, including diabetes, in many other studies in populations of mostly European origin [4, 5, 7, 9, 27]. Potential mechanisms include altered AA metabolism in the liver, kidneys, muscle or adipose tissue [29-31]. Serum creatinine as a marker of renal function did not explain associations between AAs and incident diabetes, nor did adjustment for alcohol intake, although we did not have other baseline measures of liver function. Two recent studies in European adults suggest that altered branched chain and aromatic AA metabolism is associated with impaired insulin sensitivity prior to the development of hyperglycaemia [26, 32]. South Asian individuals have lower muscle mass and more hepatic fat than Europeans [33-36]. Abdominal adipose tissue in South Asian individuals contains larger and more dysfunctional adipocytes [37]. It has also been suggested that increased AA levels may result from increased protein turnover in association with increased central obesity and reduced lean body mass [7]; this is a plausible, but untested, explanation for the increased levels of isoleucine, tyrosine, alanine and glutamine seen in our more centrally obese South Asian men.

It is not clear why associations between branched chain AAs and tyrosine and incident diabetes should be stronger in South Asian men. It is possible that this relates simply to the higher risk of baseline diabetes, but it is also possible that AA perturbations become more severe with a greater degree and/ or longer duration of insulin resistance.

It is notable that tyrosine levels were substantially higher in the South Asian men, whereas ethnic differences in other AA levels were less marked. This suggests that higher tyrosine levels may not be due solely to increased insulin resistance in South Asian individuals.

There were no significant differences in the associations between AAs and incident diabetes between the South Asian subgroups, who traditionally have different dietary practices, Hindus being mostly vegetarian and Muslims being omnivorous (non-pork) eaters, while Punjabi Sikhs have few dietary restrictions. Only five Muslim participants attended the baseline studies during Ramadan fasting. Although adjustment for self-reported dietary intake did not affect the associations between AAs and incident diabetes, studies of the associations between dietary intake and AA levels have shown large diurnal and circadian variations and highly variable individual responses to different dietary components [38-42]. Further research is needed to understand the role of diet in the relationship between serum AA levels and risk of diabetes.

Strengths and limitations This is the first population-based study on AA profiles with a large number of South Asian migrants and native Europeans conducted in the same setting and with a lengthy follow-up. However, the study was conducted only in middle-aged men and the findings may not apply to women or other age groups.

Loss to follow-up occurred in approximately one-third of the participants, and while the baseline characteristics of the whole study group and those with follow-up data only were almost identical, it is likely that those lost to follow-up would be more at risk of developing diabetes and have more adverse outcomes, leading to a possible underestimation of the associations between AAs and incident diabetes. However, loss to follow-up did not differ by ethnicity, and it is unlikely that the strengths of the associations within ethnic groups would differ in those lost to follow-up.

We did not have baseline measures of hepatic function; hence we could not explore hepatic effects on AA levels and associations.

In addition, many associations have been compared within and between ethnic groups, and although the trends are consistent with previously published results in European populations, caution is needed in interpreting multiple comparisons.

Finally, comparisons of AA associations with incident diabetes within and between South Asian subgroups are based on small numbers and should be interpreted with caution.

Summary and conclusions Higher levels of isoleucine, phenylalanine, tyrosine, alanine and glutamine were observed in South Asian men. The associations between AAs and measures of obesity were generally less marked in the South Asian men. Levels of branched chain and aromatic AAs, particularly tyrosine, were adversely associated with incident diabetes to a greater extent in South Asian than in European men, even after adjustment for previously established risk factors, including insulin resistance and obesity. These findings suggest that higher branched chain and aromatic AAs, particularly tyrosine, may be a focus for identifying novel aetiological mechanisms and potential treatment targets for diabetes in South Asian individuals and may contribute to their excess risk of diabetes.

Acknowledgements The authors are grateful to the participants for their continued support and to all members of the SABRE Study Group for their contributions to the study design, study management, data collection and analyses. 
Funding The metabolomics analyses were funded by Diabetes UK (13/ 0004774). The SABRE Study was funded at baseline by the UK Medical Research Council, Diabetes UK and the British Heart Foundation, and at follow-up by the Wellcome Trust and British Heart Foundation. QW, PW, MA-K are supported by the Academy of Finland, the Sigrid Juselius Foundation and the Strategic Research Funding from the University of Oulu, Finland. NC and ADH receive support from the National Institute for Health Research University College London Hospitals Biomedical Research Centre. NGF is supported by the Medical Research Council Epidemiology Unit (MC UU 12015/5). The SABRE Study Group is entirely independent from the funding bodies, who played no role in these analyses or in the decision to submit the manuscript for publication.

Duality of interest PW and MA-K are shareholders of Brainshake Ltd, a startup company offering NMR-based metabolite profiling. The other authors declare that there is no duality of interest associated with this manuscript.

Contribution statement TT drafted the manuscript and performed the statistical analyses. QW performed initial statistical analyses. PW and MA-K oversaw the AA quantification and contributed to the analysis and interpretation of the data, ADH, NS, NGF, IFG, PMM, SVE and $\mathrm{NC}$ contributed to the design and the acquisition of the data. All authors critically revised and approved the final manuscript. TT guarantees the work, had full access to all the data in the study and takes responsibility for the integrity of the data and the accuracy of the data analysis.

Open Access This article is distributed under the terms of the Creative Commons Attribution License which permits any use, distribution, and reproduction in any medium, provided the original author(s) and the source are credited.

\section{References}

1. Shaw JE, Sicree RA, Zimmet PZ (2010) Global estimates of the prevalence of diabetes for 2010 and 2030. Diabetes Res Clin Pract 87:4-14

2. Anand SS, Yusuf S, Vuksan V et al (2000) Differences in risk factors, atherosclerosis, and cardiovascular disease between ethnic groups in Canada: the Study of Health Assessment and Risk in Ethnic groups (SHARE). Lancet 356:279-284

3. Tillin T, Hughes AD, Godsland IF et al (2013) Insulin resistance and truncal obesity as important determinants of the greater incidence of diabetes in Indian Asians and African Caribbeans compared with Europeans: the Southall And Brent REvisited (SABRE) cohort. Diabetes Care 36:383-393

4. Newgard CB, An J, Bain JR et al (2009) A branched-chain amino acid-related metabolic signature that differentiates obese and lean humans and contributes to insulin resistance. Cell Metab 9:311-326

5. Wang TJ, Larson MG, Vasan RS et al (2011) Metabolite profiles and the risk of developing diabetes. Nat Med 17:448-453

6. Magnusson M, Lewis GD, Ericson U et al (2012) A diabetespredictive amino acid score and future cardiovascular disease. Eur Heart J 34:1982-1989

7. Tai ES, Tan ML, Stevens RD et al (2010) Insulin resistance is associated with a metabolic profile of altered protein metabolism in Chinese and Asian-Indian men. Diabetologia 53:757-767

8. Floegel A, Stefan N, Yu Z et al (2012) Identification of serum metabolites associated with risk of type 2 diabetes using a targeted metabolomic approach. Diabetes 62:639-648
9. Huffman KM, Shah SH, Stevens RD et al (2009) Relationships between circulating metabolic intermediates and insulin action in overweight to obese, inactive men and women. Diabetes Care 32:16781683

10. Stancakova A, Civelek M, Saleem NK et al (2012) Hyperglycemia and a common variant of GCKR are associated with the levels of eight amino acids in 9,369 Finnish men. Diabetes 61:1895-1902

11. Gogna N, Krishna M, Oommen AM, Dorai K (2015) Investigating correlations in the altered metabolic profiles of obese and diabetic subjects in a South Indian Asian population using an NMR-based metabolomic approach. Mol Biosyst 11:595-606

12. Tillin T, Forouhi NG, McKeigue PM, Chaturvedi N (2010) Southall And Brent REvisited: Cohort profile of SABRE, a UK populationbased comparison of cardiovascular disease and diabetes in people of European, Indian Asian and African Caribbean origins. Int $\mathrm{J}$ Epidemiol 41:33-42

13. Baecke JA, Burema J, Frijters JE (1982) A short questionnaire for the measurement of habitual physical activity in epidemiological studies. Am J Clin Nutr 36:936-942

14. World Health Organization (1999) Definition, diagnosis and classification of diabetes mellitus and its complications. Part 1: diagnosis and classification of diabetes mellitus. WHO, Geneva

15. Levy JC, Matthews DR, Hermans MP (1998) Correct homeostasis model assessment (HOMA) evaluation uses the computer program. Diabetes Care 21:2191-2192

16. Matsuda M, DeFronzo RA (1999) Insulin sensitivity indices obtained from oral glucose tolerance testing: comparison with the euglycemic insulin clamp. Diabetes Care 22:1462-1470

17. DeFronzo RA, Matsuda M (2010) Reduced time points to calculate the composite index. Diabetes Care 33:e93

18. Soininen P, Kangas AJ, Wurtz P et al (2009) High-throughput serum NMR metabonomics for cost-effective holistic studies on systemic metabolism. Analyst 134:1781-1785

19. Kettunen J, Tukiainen T, Sarin AP et al (2012) Genome-wide association study identifies multiple loci influencing human serum metabolite levels. Nat Genet 44:269-276

20. Inouye M, Kettunen J, Soininen P et al (2010) Metabonomic, transcriptomic, and genomic variation of a population cohort. Mol Syst Biol 6:441

21. Fisher R (1921) On the probable error of a coefficient deduced from a small sample. Metron 1:3-32

22. Morgan MY, Marshall AW, Milsom JP, Sherlock S (1982) Plasma amino-acid patterns in liver disease. Gut 23:362-370

23. Newsom R (2010) Comparing the predictive powers of survival models using Harrell's C or Somers' D. Stata J 10:339-358

24. Pencina MJ, Agostino RBD, Agostino RBD, Vasan RS (2008) Evaluating the added predictive ability of a new marker: from area under the ROC curve to reclassification and beyond. Stat Med 27:157-172

25. Fine JP, Gray R (1999) A proportional hazards model for the subdistribution of a competing risk. J Am Stat Assoc 94:496-509

26. Wurtz P, Soininen P, Kangas AJ et al (2013) Branched-chain and aromatic amino acids are predictors of insulin resistance in young adults. Diabetes Care 36:648-655

27. Fiehn O, Garvey WT, Newman JW, Lok KH, Hoppel CL, Adams SH (2010) Plasma metabolomic profiles reflective of glucose homeostasis in non-diabetic and type 2 diabetic obese African-American women. PLoS ONE 5:e15234

28. Persson M, Nilsson JA, Nelson JJ, Hedblad B, Berglund G (2007) The epidemiology of Lp-PLA(2): distribution and correlation with cardiovascular risk factors in a population-based cohort. Atherosclerosis 190:388-396

29. Krebs M, Krssak M, Bernroider E et al (2002) Mechanism of amino acid-induced skeletal muscle insulin resistance in humans. Diabetes 51:599-605 
30. She P, Van HC, Reid T, Hutson SM, Cooney RN, Lynch CJ (2007) Obesity-related elevations in plasma leucine are associated with alterations in enzymes involved in branched-chain amino acid metabolism. Am J Physiol Endocrinol Metab 293:E1552-E1563

31. Tremblay F, Brule S, Hee US et al (2007) Identification of IRS-1 Ser1101 as a target of S6K1 in nutrient- and obesity-induced insulin resistance. Proc Natl Acad Sci U S A 104:14056-14061

32. Wurtz P, Tiainen M, Makinen VP et al (2012) Circulating metabolite predictors of glycemia in middle-aged men and women. Diabetes Care 35:1749-1756

33. Petersen KF, Dufour S, Feng J et al (2006) Increased prevalence of insulin resistance and nonalcoholic fatty liver disease in Asian-Indian men. Proc Natl Acad Sci U S A 103:18273-18277

34. Anand SS, Tarnopolsky MA, Rashid S et al (2011) Adipocyte hypertrophy, fatty liver and metabolic risk factors in South Asians: the Molecular Study of Health and Risk in Ethnic Groups (molSHARE). PLOS ONE 6:e22112

35. Eastwood SV, Tillin T, Wright A et al (2014) Thigh fat and muscle each contribute to excess cardiometabolic risk in South Asians, independent of visceral adipose tissue. Obesity (Silver Spring) 22:20712079

36. Chowdhury B, Lantz H, Sjostrom L (1996) Computed tomographydetermined body composition in relation to cardiovascular risk factors in Indian and matched Swedish males. Metabolism 45: 634-644

37. Chandalia M, Lin P, Seenivasan T et al (2007) Insulin resistance and body fat distribution in South Asian men compared to Caucasian men. PLoS ONE 2:e812

38. Fernstrom JD, Wurtman RJ, Hammarstrom-Wiklund B, Rand WM, Munro HN, Davidson CS (1979) Diurnal variations in plasma concentrations of tryptophan, tyrosine, and other neutral amino acids: effect of dietary protein intake. Am J Clin Nutr 32:1912-1922

39. Weller LA, Margen S, Calloway DH (1969) Variation in fasting and postprandial amino acids of men fed adequate or protein-free diets. Am J Clin Nutr 22:1577-1583

40. Nasset ES, Heald FP, Calloway DH, Margen S, Schneeman P (1979) Amino acids in human blood plasma after single meals of meat, oil, sucrose and whiskey. J Nutr 109:621-630

41. Riggio O, Merli M, Pieche U et al (1989) Circadian rhythmicity of plasma amino acid variations in healthy subjects. Recenti Prog Med 80:591-593

42. Ashley DV, Barclay DV, Chauffard FA, Moennoz D, Leathwood PD (1982) Plasma amino acid responses in humans to evening meals of differing nutritional composition. Am J Clin Nutr 36:143-153 